\title{
Evaluation of Texture Features for Analysis of Ovarian Follicular Development
}

\author{
Na Bian ${ }^{1}$, Mark. G. Eramian ${ }^{1}$, and Roger A. Pierson ${ }^{2}$ \\ ${ }^{1}$ Department of Computer Science, University of Saskatchewan \\ n.bian@usask.ca, eramian@cs.usask.ca \\ ${ }^{2}$ Department of Obstetrics, Gynecology and Reproductive Sciences, \\ University of Saskatchewan \\ pierson@erato.usask.ca
}

\begin{abstract}
We examined the echotexture in ultrasonographic images of the wall of dominant ovulatory follicles in women during natural menstrual cycles and dominant anovulatory follicles which developed in women using oral contraceptives (OC). Ovarian follicles in women are fluid-filled structures in the ovary that contain oocytes (eggs). Dominant follicles are physiologically selected for preferential development and ovulation. Statistically significant differences between the two classes of follicles were observed for two co-occurrence matrix derived texture features and two edge-frequency based texture features which allowed accurate distinction of healthy and atretic follicles of similar diameters. Trend analysis revealed consistent turning points in time series of texture features between 3 and 4 days prior to ovulation coinciding with the time at which follicles are being biologically "prepared" for ovulation.
\end{abstract}

\section{Introduction}

Ovarian follicles are fluid-filled structures in human ovaries which contain the oocytes (eggs). Early studies of follicular activity have relied on measurement of reproductively active hormones; Leutenizing Hormone (LH), follicle stimulating hormone (FSH), estradiol (E2), and progesterone [6, 11]. Recent studies [1, 3, 5] have used high resolution transvaginal ultrasonography to visualize follicular activity. Ultrasonography is non-invasive and permits sequential analyses, therefore broadening research approaches to the study of folliculogenesis.

A normal dominant follicle is destined to ovulate and produces increasing amounts of E2 as it develops. In the final phase of growth, a rapid increase in E2 levels trigger an acute increase of $\mathrm{LH}$ that causes ovulation approximately $38 \mathrm{~h}$ later. Subsequently, the collapsed follicle undergoes structural and functional transformation to become the corpus luteum. Peak E2 concentrations occur on the day of, or one day prior to, ovulation [1].

Exploration of follicular development during oral contraceptive (OC) use is a current area of interest. Dominant follicles grow to pre-ovulatory sizes under the influence of OC but most do not ovulate [3. It is difficult to visually differentiate ovulatory follicles in natural menstrual cycles from anovulatory follicles in OC users with ultrasonography although the hormonal milieu is quite different. 


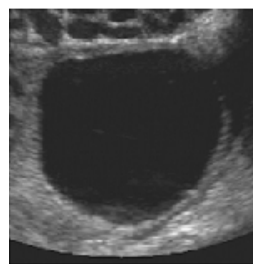

(a)

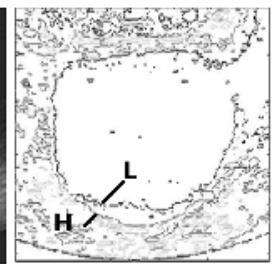

(b)

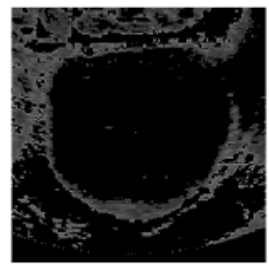

(c)

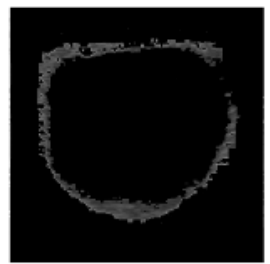

(d)

Fig. 1. Selection of follicle wall. (a) Original image, (b) isocontours of (a), (c) pixels with intensity between selected contours $\mathrm{H}$ and $\mathrm{L}$, (d) homogeneous follicle wall region segmented from (c) using EDISON [7].

The purpose of our study was to identify texture features which can be used to 1) distinguish between dominant follicles in women during natural cycles and women using OC, and 2) determine when during their development these two classes of follicles begin to exhibit differences.

\section{Materials and Methods}

Our data set consisted of 15 temporal series of ultrasonographic images of dominant follicles acquired on a semi-daily schedule. Eight series were from women with natural menstrual cycles [1] and seven were from women using OC [2]. Each series from natural cycles contained six to eight images beginning 7 days prior to ovulation and ending on the day of ovulation. Each series from OC cycles consisted of 3 to 8 images beginning 7 days before the E2 peak. The OC series were aligned with the natural cycle series by synchronizing E2 peaks with the days of ovulation. All images were acquired using the same equipment and techniques [1, 3]. In total, there were 57 images in the natural cycle data set and 34 images in the $\mathrm{OC}$ cycle data set. All images were $640 \times 480$ pixel arrays with a dynamic range of 256 gray levels.

The fluid-filled interior of follicles exhibited echo-responses close to the level of background noise (the dark area in Fig. 1(a)). Therefore we attempted to measure differences the echotexture of the follicle walls. The follicle wall is a $1-2 \mathrm{~mm}$ thick area surrounding the fluid-filled interior. Our method consisted of preprocessing stages which included selection of the follicle wall regions and greylevel adjustment, followed by computation of features and their analysis.

\section{Preprocessing}

\subsection{Follicle Wall Selection}

Manual and automatic contouring were used to select follicle wall regions. Isocontours were computed and contours corresponding to the approximate inner and outer edges of the follicle wall were manually selected (e.g. contours $H$ and 
$L$ in Figure 1(b)). The greylevels of the contours were used as upper and lower thresholds for semi-band-thresholding of the original image (Figure 1(c)).

Figure 1(d) was obtained from that in Figure 1(c) using the EDISON System v1.1 (Edge Detection and Image SegmentatiON) 7] which used user-defined thresholds to segment the original image into homogeneous regions with respect to mean intensity. The thresholds used to segment each image were manually selected and differed from image to image.

The resulting follicle wall images were black in background while the gray levels of follicle walls remained unchanged. The regions obtained were not always contiguous because portions of the follicle wall become very thin at the apex as the interval to ovulation decreases. This does not present a problem when computing features as one simply excludes zero-intensity pixels.

\subsection{Gray Level Adjustment}

We subtracted from each pixel the mean intensity of a $3 \times 3$ square neighborhood about the pixel, following the preprocessing procedure from [4]. Though the authors of [4] used an $11 \times 11$ neighborhood, we used $3 \times 3$ since our regions of interest were only a few pixels wide. Background pixels were not considered when they appeared within the neighborhood of a pixel and did not contribute to the average. The resulting pixel values were shifted into the interval $[0, M]$ using an additive constant and then normalized into the interval $[0,255]$.

\section{Extraction of Texture Features}

We chose to investigate only directionally invariant features since it is not anatomically possible monitor each patient in the same orientation consistently. Eight texture features, described in the following subsections, were computed for each follicle wall image.

\subsection{Co-occurrence Features}

Haralick's Graylevel co-occurrence matrices (GLCM) [8] describe the joint graylevel histogram of the image. Homogeneity, correlation, contrast and energy were chosen from the 14 features that Haralick defined. A displacement distance of one pixel was selected since our regions of interest were, in some places, only a few pixels wide. The four symmetric co-occurrence matrices for the $0^{\circ}, 45^{\circ}$, $90^{\circ}$, and $135^{\circ}$ directions were computed. Prior to normalizing the matrices, the first row and column of each matrix were replaced with zeros to eliminate cooccurrences between foreground and background pixels. The resulting matrices were normalized and the four texture features computed from each matrix. For each feature, the four values from each direction were averaged to obtain four rotationally invariant features for each image. 


\subsection{Edge Frequency Features}

We computed edge density and edge contrast as representatives of texture features based on edge frequency measures [14. Edge contrast is the average edge magnitude of a region where edge magnitudes were calculated using a $3 \times 3$ Laplacian operator.

Edge density is the proportion of pixels with "strong" gradient magnitudes. We approximated the gradient as a function of distance $d$. Let $M$ and $N$ be pixel dimensions of the image $I$. The gradient magnitude image $g_{d}$ of $I(i, j)$ given distance $d$ is:

$$
\begin{gathered}
g_{d}(i, j)=|I(i, j)-I(i+d, j)|+|I(i, j)-I(i-d, j)|+ \\
\\
|I(i, j)-I(i, j+d)|+|I(i, j)-I(i, j-d)| .
\end{gathered}
$$

The edge density texture descriptor $g(d)$ is the mean of $g_{d}(i, j)$. Our analysis proceeds with $d=1$. This scheme avoided the problem of selecting appropriate thresholds for measuring proportion of pixels with "strong" gradient magnitude.

\subsection{Laws' Measures}

Laws' measures compute a set of energy statistics for each pixel in a texture image from which global texture features are derived. We computed the features $E 5 L 5, L 5 E 5, E 5 S 5$ and $S 5 E 5$, as denoted by Laws 10 . To obtain rotationally invariant features the symmetric energy measures were combined, via averaging, to obtain two features: $E 5 L 5 / L 5 E 5$ and $E 5 S 5 / S 5 E 5$.

\section{Selection of Discriminator Features}

A series of experiments were carried out to determine if any of our candidate features were good discriminators of the two classes of follicles.

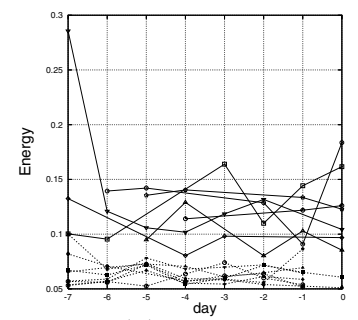

(a) energy

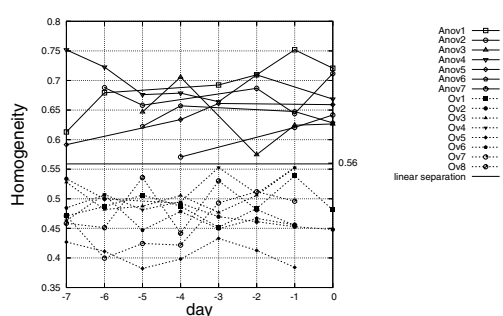

(b) homogeneity

Fig. 2. Co-occurrence features of follicle wall images. Anov1 to Anov7 were from OC cycle group, Ov1 to Ov8 were from the natural cycle group.

Figures 2 and 3 represent computed texture feature values for each of our 15 image series. The horizontal and vertical axes, respectively, denote cycle day 


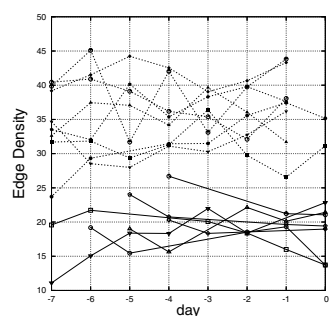

(a) edge density

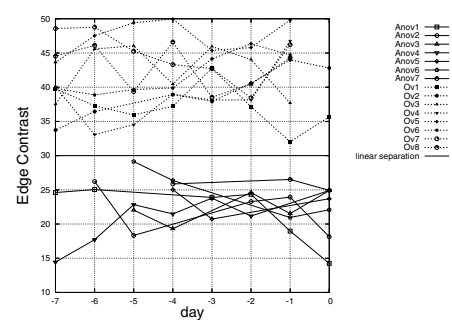

(b) edge contrast

Fig. 3. Edge frequency features of follicle wall images. Anov1 to Anov7 were from the OC cycle group, Ov1 to Ov8 were from the natural cycle group.

(where day 0 is day of ovulation/E2 peak) and texture feature value. Figures 2(a) and 2(b), show that the images could be separated into two categories in the GLCM energy and homogeneity feature spaces since the feature values from the different classes form distinct populations $(p<0.0001)$. This indicates that the features are likely to be powerful features for class discrimination. Similarly, Figure 3 shows the computed edge contrast and edge density feature values. Follicle wall images in the natural menstrual cycles had consistently higher edge density and edge contrast $(p<0.001)$ indicating that these are also discriminating features.

GLCM contrast and correlation, E5S5/S5E5 and E5L5/L5E5 measures exhibited considerable overlap between the two populations $(p<0.04)$ and were considered to be inferior class discriminators compared to the others.

\section{Classification Using a Single Texture Feature}

We investigated the ability of each feature to classify our image series as belonging to either a natural cycle or an $\mathrm{OC}$ cycle. For each series and texture feature, a vector was produced consisting of the measured values of that feature on each day. All feature vectors were of size 8 where missing data due to lack of an image on a particular day in a series was generated by linear interpolation of neighboring samples. For each feature, six randomly chosen vectors (3 normal and 3 OC) were used as training data. Remaining vectors were used for testing.

To classify the test set, we fit a multivariate normal density to each data set such that the difference of the means in the two groups was maximum and each observation in the testing samples was assigned to its nearest group [12. MATLAB 7's classify() function was used to perform the classification; rates are shown in Table 1 Edge density, edge contrast, GLCM homogeneity and GLCM energy were able to correctly classify the test set with $100 \%$ accuracy. Both Laws' energy statistics had a classification rate of $77.8 \%$ while cooccurrence contrast and GLCM correlation achieved rates of $66.7 \%$ and $44.4 \%$, respectively. 
Table 1. Single-feature classification rate of ovulatory and anovulatory follicles

\begin{tabular}{|l|c||l|c|}
\hline Feature & \% Correct & Feature & \% Correct \\
\hline \hline Edge Density & $100 \%$ & GLCM Energy & $100 \%$ \\
\hline Edge Contrast & $100 \%$ & GLCM Homogeneity & $100 \%$ \\
\hline Laws' E5S5/S5E5 & $77.8 \%$ & GLCM Contrast & $66.7 \%$ \\
\hline Laws' E5L5/L5S5 & $77.8 \%$ & GLCM Correlation & $44.4 \%$ \\
\hline
\end{tabular}

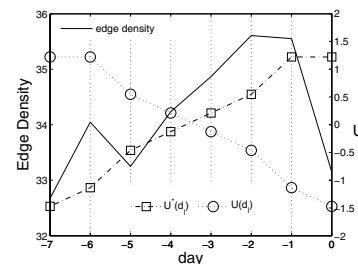

(a)

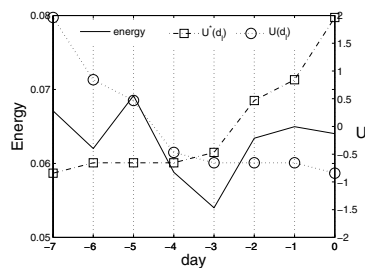

(c)

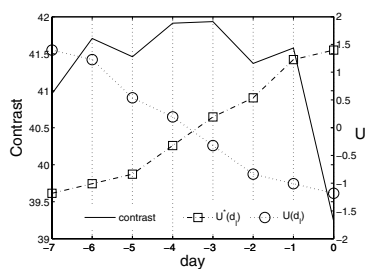

(b)

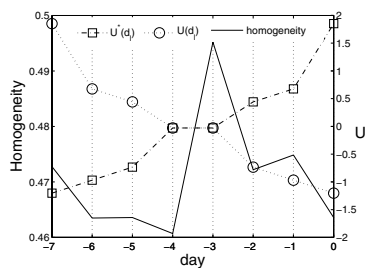

(d)

Fig. 4. Turning points for (a) edge density, (b) edge contrast, (c) GLCM energy, and (d) GLCM homogeneity of ovulatory follicle images

\section{Trend Analysis Using the Mann-Kendall Method}

The Mann-Kendall method was used to locate changes in the trends (upward or downward) of feature values over time. The method used by Sneyers [13] and Johnson et. al. 9] was followed which estimates the single turning point (change of trend) of a series:

1. Given a sequence of feature values $f_{t}, t=1,2, \ldots, l, l \leq N$, the test statistic is $d_{l}=\sum_{i<j} \operatorname{sign}\left(f_{j}-f_{i}\right)$ where $\operatorname{sign}(x)=1$ if $x$ is positive, and 0 otherwise.

2. Let $E\left(d_{l}\right)$ and $\operatorname{Var}\left(d_{l}\right)$ be the expected value and variance of $d_{l}, l=1,2, \ldots, N$. Calculate the $N$ statistics $U\left(d_{l}\right)$ where

$$
U\left(d_{l}\right)=\left(d_{l}-E\left(d_{l}\right)\right) / \sqrt{\operatorname{Var}\left(d_{l}\right)} .
$$

3. Repeat 1 and 2 on the original sequence in reverse order to obtain $U_{\text {new }}\left(d_{l}\right)$. Let $U^{*}\left(d_{l}\right)=-U_{\text {new }}\left(d_{N-l+1}\right)$. Plot $U^{*}\left(d_{l}\right)$ and $U\left(d_{l}\right)$ on the same axes.

4. If $|U| \leq 1.96$ at the time of intersection, $x$, of the two plots, then Mann's test supports the hypothesis of a turning point at time $x$ with $95 \%$ confidence. 


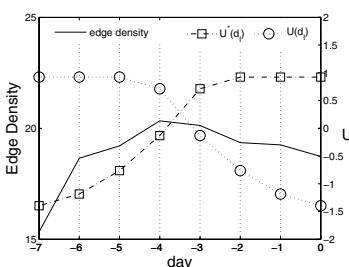

(a)

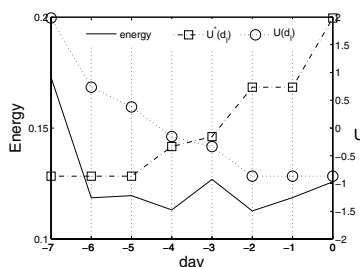

(c)

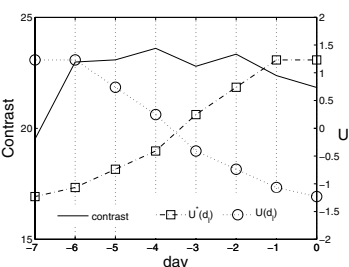

(b)

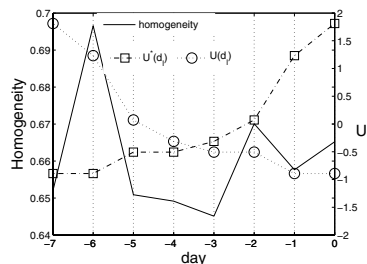

(d)

Fig. 5. Turning points for (a) edge density, (b) edge contrast, (c) GLCM energy, and (d) GLCM homogeneity of anovulatory follicle images

The application of the Mann-Kendall method on both the natural cycle group and $\mathrm{OC}$ cycle group are depicted in Figures 4 and 5, respectively. The solid curves represent the average feature value on each day over all women in their respective group. The dotted curves are the statistics $U$ and $U^{*}$, respectively. On each plot, there is one point of intersection of $U$ and $U^{*}$ such that $|U| \leq 1.96$, namely, the turning point. The observation of these turning points was interpreted as an indication of a physiologic change in follicle walls associated with impending ovulation occurring 3 or 4 days before ovulation, or E2 peak, respectively.

\section{Conclusion and Discussion}

We discovered that it is possible to distinguish dominant follicles during natural cycles from those in women using OC though in vivo ultrasonography. GLCM energy, GLCM homogeneity, edge density and edge contrast texture measures of the follicle walls of anovulatory dominant follicles during $\mathrm{OC}$ cycles were significantly different than those of ovulatory dominant follicles from women in natural menstrual cycles. Moreover, all four features were able to classify images as either normal or OC-user series with $100 \%$ accuracy.

Lower homogeneity observed in ovulatory follicles was attributed to increased vascularity and interstitial edema as ovulation approached. Lower edge density and contrast values observed in anovulatory follicles indicated coarser textures on the follicle walls. This was attributed to failure to develop interstitial edema and peripheral vascularity.

Turning points for edge density, edge contrast, GLCM homogeneity and GLCM energy were found around the 3rd or 4th day before ovulation for ovulatory follicles and around the 3rd or 4th day before peak E2 for anovulatory follicles. This 
is an exciting observation as it coincides with the time at which follicles are being biologically "prepared" for ovulation. This coincidence suggests that texture features of ultrasound images could be used to detect physiologic changes associated with impending ovulation which would be a useful aid to oocyte harvesting for in vitro fertilization.

Future work will investigate the question of whether a similar approach could be used to identify other biological events such as physiologic selection of dominant follicles.

\section{References}

[1] A. R. Baerwald, G. P. Adams, and R. A. Pierson. Characterization of ovarian follicular wave dynamics in women. Biology of Reproduction, 69(1):1023-1031, 2003.

[2] A. R. Baerwald, O. A. Olatunbosun, and R. A. Pierson. Ovarian follicular waves are initiated during the hormone free interval in women using oral contracption. Contraception, 70(5):371-377, 2004.

[3] A. R. Baerwald and R. A. Pierson. Ovarian follicular development during the use of oral contraception: A review. Journal of Obstetrics and Gynaecology Canada, 26(1):19-24, Jan 2004.

[4] O. Basset, Z. Sun, J. L. Mestas, and G. Gimenez. Texture analysis of ultrasonic images of the prostate by means of co-occurrence matrices. Ultrasonic Imaging, 15:218-237, 1993.

[5] M. Broome, J. Clayton, and K. Fotherby. Enlarged follicles in women using oral contraceptives. Contraception, 52:13-16, 1995.

[6] P. G. Crosignani, G. Testa, W. Vegetti, and F. Parazzini. Ovarian activity during regular oral contraceptive use. Contraception, 24(5):271-273, 1996.

[7] B. Georgescu and C. M. Christoudias. Code for the edge detection and image segmentation system. http://www.caip.rutgers.edu/riul/research/code/ EDISON/index.html.

[8] R. M. Haralick and K. Shanmugam. Textural feature for image classification. IEEE Transactions On Systems, Man, And Cybernetics, SMC-3(6):610-619, November 1980.

[9] N. L. Johnson, C. B. Read, and S. Kotz. Encyclopedia of Statistical Sciences. New York : Wiley, 1981.

[10] K. I. Laws. Texture energy measures. Proc.Image Understanding Workshop, pages 41-51, 1979.

[11] M. A. Renier, A. Vereechen, E. V. Herck, D. Straetmans, P. Ramaekers, J. Vanderheyden, H. Degezelle, and P. Buytaert. Dimeric inhibin serum values as markers of ovarian activity in pill-free intervals. Contraception, 57(1):45-48, January 1998.

[12] G. A. F. Seber. Multivariate Observations. Wiley Series in Probability and Mathematical Statistics. Wiley, 1984.

[13] R. Sneyers. On the statistical analysis of series of observations. Technical note, World Meteorological Organization Technical Note, 1990.

[14] M. Sonka, V. Hlavac, and R. Boyle. Image Processing, Analysis and Machine Vision, chapter 14, pages 653-656. Brooks/Cole, A Division of Thomson Asia Pte Led, USA, 2 edition, 1998. 